\title{
Resultados, aportes e impactos tras ocho años del desarrollo de proyectos forestales por parte del INISEFOR en la zona sur de Costa Rica
}

\section{Results, Contributions, and Impacts After Eight Years of Forestry Projects Developed by INISEFOR in the Southern Region of Costa Rica}

Ana Isabel Barquero Elizondo

Universidad Nacional

Heredia, Costa Rica ana.barquero.elizondo@una.cr

Carlos Enrique Ávila Arias

Universidad Nacional

Heredia, Costa Rica carlos.avila.arias@una.cr

William Hernández Castro

Universidad Nacional

Heredia, Costa Rica

william.hernandez.castro@una.cr

Rafael Murillo Cruz

Universidad Nacional

Heredia, Costa Rica

rafael.murillo.cruz@una.cr

Recibido: 13/02/2018 Aceptado: 15/05/2018

Resumen. El trabajo realizado por el Instituto de Investigación y Servicios Forestales (INISEFOR) de la Universidad Nacional (UNA) en varios cantones de la Zona Sur de Costa Rica muestra cómo los resultados de la investigación, combinados con la 
Revista Universidad en DiÁlogo • Vol. 8, N. ², Julio-Diciembre, 2018, pp. 11-23

ISSN 2215-2849 • EISSN: 2215-4752

DOI: http://dx.doi.org/10.15359/udre.8-2.1

docencia y la producción, se constituyen en la base para un proceso de extensión universitaria de largo plazo. Se recabó datos sobre las actividades productivas relacionadas con el agro y sobre la cadena de valor forestal, compuesta por varios eslabones en los cuales se integran más de ciento cincuenta actores directos, en los cantones de Osa y Golfito. Debido a la necesidad de proveer a los productores de materiales genéticos adecuados, se estableció un vivero de reproducción vegetativa y plantaciones clonales piloto, las cuales además de servir de campos demostrativos para los finqueros, cumplen una importante función docente en la formación de los profesionales forestales de la UNA. Debido a las características propias de la actividad forestal, la extensión universitaria en este campo requiere de un abordaje interdisciplinario y debe ser programada a largo plazo, pues los árboles necesitan de un período mayor de tiempo para su crecimiento y posterior cosecha y los pobladores involucrados necesitan complementariedad con otras actividades productivas, a fin de lograr un verdadero impacto positivo en sus condiciones de vida.

Palabras claves: Gmelina arborea, reforestación, desarrollo sostenible, Tectona grandis, extensión universitaria.

\begin{abstract}
This paper presents an account of the work that the Universidad Nacional carried out in the forestry field in several counties of the southern part of Costa Rica. The paper aims to visualize how the results of the research, combined with teaching and production, constitute the base for a long-term university extension process. It was possible to collect data about the agricultural activities in the area and the existence of a forest value chain composed of several links; in these links, more than one hundred and fifty direct actors are integrated, only in the counties of Osa and Golfito. Due to the need for forest producers to have adequate genetic materials, a nursery and pilot plantations were established. The plantations work as demonstration fields for the farmers and play an important educational role in the training of forestry students of the Universidad Nacional. Due to the characteristics of the forestry activity, in this field university extension processes require an interdisciplinary approach and must be programmed in the long term, since trees need a more extended period for their growth and subsequent harvest, and the producers involved need to have complementarity with other activities, in order to achieve better living conditions.
\end{abstract}

Keywords: Gmelina arborea, reforestation, sustainable development, Tectona grandis, university extension. 


\section{Introducción}

Desde el año 2007, el INISEFOR lleva a cabo proyectos forestales en la Zona Sur de Costa Rica, los cuales han logrado una serie de impactos en diversas comunidades, principalmente de los cantones de Pérez Zeledón (provincia de San José), Osa, Corredores y Golfito (provincia de Puntarenas). Estas zonas se encuentran entre las de menor índice de desarrollo social del país, ocupando las posiciones 47, 69, 74 y 77 respectivamente, entre los 81 cantones (Mideplan, 2013).

Tanto por las condiciones fisiográficas, bioclimáticas y de enclave turístico que posee la región, como porque en la misma confluyen las distintas personas involucradas que componen la cadena productiva forestal (desde la producción en vivero del material a plantar y las plantaciones, hasta la fase de aprovechamiento, transformación y comercialización de los productos de madera), el sector forestal podría constituirse en una alternativa interesante para esta región del país, si de previo se logra definir una serie de aspectos complementarios que le brinden sostenibilidad y de ese modo crear y promover una cultura forestal propia de la zona. Uno de los aspectos complementarios que deberá tomarse en cuenta para asegurar un futuro competitivo para estas actividades es aumentar el valor agregado de los productos o servicios, debido a la distante ubicación de estos cantones respecto de los centros de mayor población, lo que da un alto valor de transporte de las mercancías para hacerlas llegar a los mercados del Valle Central.

Se han establecido plantaciones con materiales genéticos de alta calidad, que han sido plantados en las fincas de pequeños/as y medianos/as propietarios/as, quienes han comprobado que la producción de madera es posible en sus fincas, en combinación con otros cultivos agrícolas e inclusive con la ganadería. En este documento se reúnen las experiencias y se presenta el trabajo realizado, pero también se visualiza el impacto que la extensión y la investigación universitaria logran en las comunidades rurales del país. Asimismo, se busca evidenciar el potencial de la actividad forestal como una opción productiva interesante y la necesidad de llevar a cabo un trabajo interdisciplinario, con miras a que la acción sustantiva de la Universidad cumpla con sus objetivos en las zonas menos privilegiadas del país. 
Revista Universidad en DiÁlogo • Vol. 8, N. ², Julio-Diciembre, 2018, pp. 11-23

ISSN 2215-2849 • EISSN: 2215-4752

DOI: http://dx.doi.org/10.15359/udre.8-2.1

\section{Metodología}

El INISEFOR, a través de diversos fondos de cooperación, ha ejecutado proyectos integrados en esta zona, mediante los cuales se pretende impulsar la creación de sitios de producción forestal continua, en una de las zonas de mayor potencial para este tipo de actividad, en comunidades con índices de desarrollo social de los más bajos del país, entendiendo por desarrollo social, de acuerdo con Mideplan (2013):

El proceso que en el transcurso del tiempo, procura alcanzar una mayor calidad de vida de la población, mediante una sociedad más igualitaria, participativa e inclusiva, que garantice una reducción en la brecha que existe en los niveles de bienestar que presentan los diversos grupos sociales y áreas geográficas, para lograr una integración de toda la población a la vida económica, social, política y cultural del país, en un marco de respeto y promoción de los derechos humanos. (p. 1)

En la figura 1 se visualiza la ubicación espacial de algunas de las fincas involucradas, en los cantones de Osa, Corredores y Golfito. En lo que respecta a Pérez Zeledón, se trabajó con habitantes del distrito de Pejibaye, con apoyo internacional del Fondo de Desarrollo Noruego (FDN).

\section{Sinopsis del proceso y algunos resultados obtenidos}

Diversos factores favorecieron la investigación-extensión en la Zona Sur del país, dentro de los que se destacan que cuenta con las condiciones microclimáticas adecuadas para el crecimiento exitoso de varias especies forestales; la posibilidad de incidir positivamente en algunas de las zonas socioeconómicamente marginadas del país; un sistema de financiamiento de la educación pública universitaria costarricense que permite destinar fondos a la ejecución de proyectos; $y$, finalmente, el empeño de un grupo de académicos/as y productores/as dispuestos/as a trabajar en conjunto por el desarrollo de la región.

Como punto de partida, mediante consulta de personas expertas, se analizaron diversos sistemas agrícolas de producción en la zona (ganadería, arroz, palma, plátano y melina), a fin de establecer su rendimiento en términos financieros por área de superficie, así como los factores positivos 
y negativos en su contexto socioeconómico. Los resultados muestran que la siembra de melina en sitios adecuados y usando turnos de seis a siete años es atractiva para los pobladores y las pobladoras de los cantones incluidos en el estudio; sus principales factores positivos son el bajo riesgo de pérdida del producto, su bajo impacto ambiental, dado su reducido uso de insumos agroquímicos, la generación de empleo, el alto valor agregado de la madera, entre otros (ver tabla 1). Es necesario un aumento en la cantidad de personas de estas comunidades que dan valor agregado a la madera, ya que la mayoría han sido foráneas a los cantones involucrados y esa experiencia se distancia del área de interés, lo cual podría resolverse con un proceso de educación en el sector técnico.

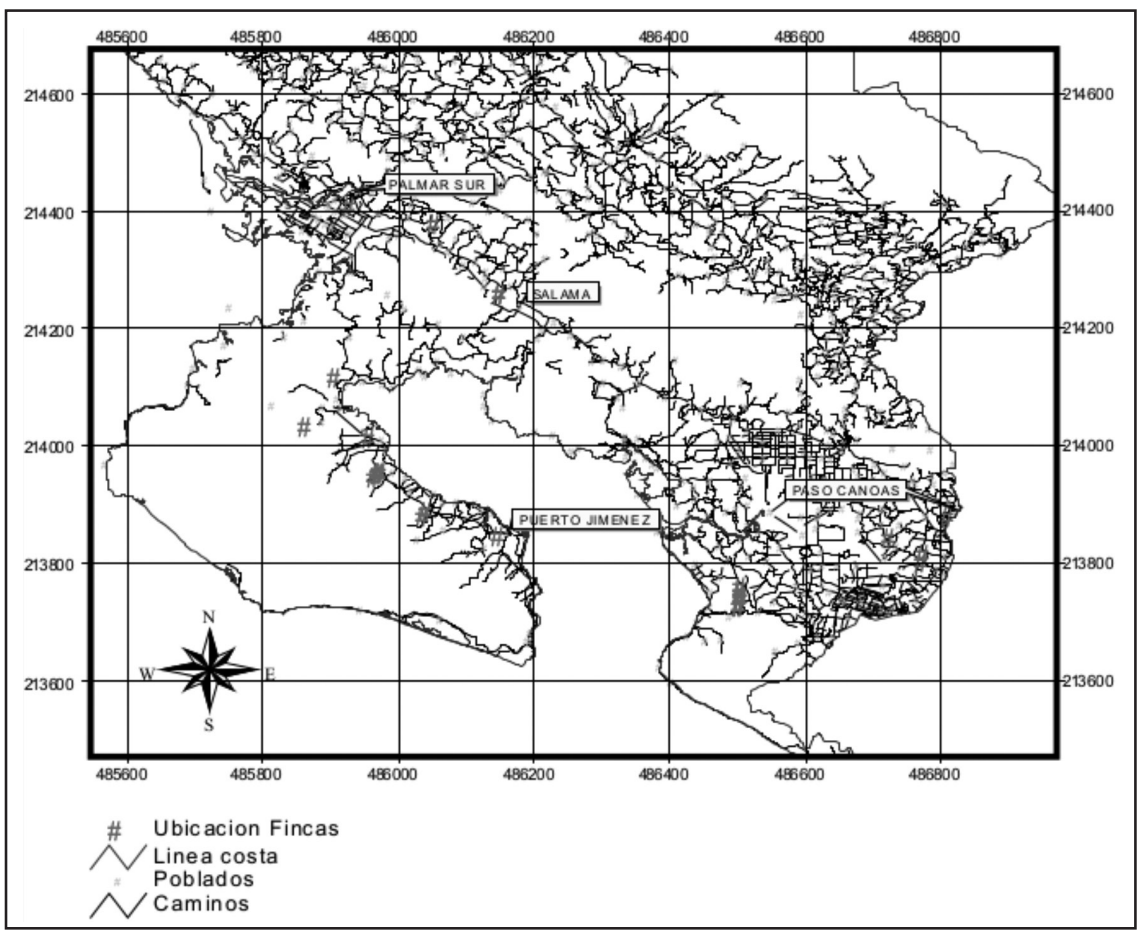

Figura 1. Ubicación de las fincas de las personas agricultoras asociadas a los proyectos. Fuente: Murillo y Ávila (2011) 
Revista Universidad en DiÁlogo • Vol. 8, N. ², Julio-Diciembre, 2018, pp. 11-23

ISSN 2215-2849 • EISSN: 2215-4752

DOI: http://dx.doi.org/10.15359/udre.8-2.1

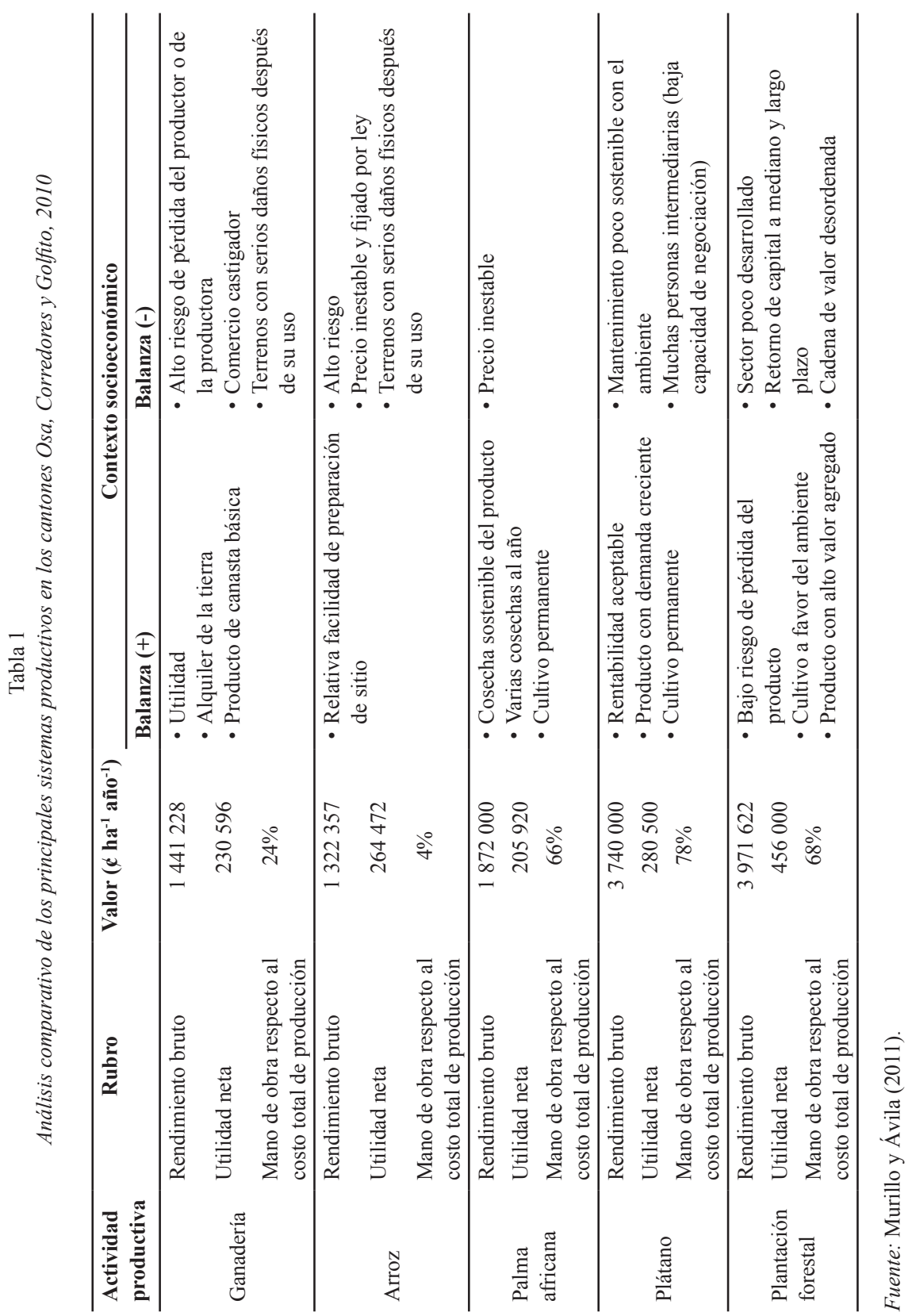


Asimismo, se llevó a cabo un estudio mediante visitas y encuestas para conocer la cadena de valor forestal, el cual permitió identificar los eslabones que la componen: viveros forestales, plantaciones forestales, bosques, aserraderos, fábricas de tarimas, fábricas de papel, cartón, pulpa, paneles, plywood, fábricas de muebles, entre otros. Adicionalmente, se identificaron canales de comercialización como depósitos, almacenes, ferreterías, salas de exhibición y todos los medios de transporte que se ven involucrados dentro del sistema (ver tabla 2).

En el 2011 la actividad forestal generaba aproximadamente 144 empleos en Osa y Golfito, de los cuales un 59\% están relacionados con las actividades de aprovechamiento y fabricación de tarimas o pallets. A través de las encuestas se determinó que existía una demanda de madera de 179000 $\mathrm{pmt}^{1}$ semanales para abastecer las industrias tarimeras y los aserraderos, principalmente de la especie melina, con alguna proporción de pino, eucalipto, teca y nativas (Murillo y Ávila, 2014).

\section{Tabla 2}

Número de personas involucradas según eslabón de la cadena de valor forestal en Osa (2010) y Golfito (2011)

\begin{tabular}{lccr}
\hline Actividad & $\mathbf{N}^{\circ}$ de personas Osa & $\mathbf{N}^{\circ}$ de personas Golfito & Total \\
\hline Vivero & 4 & 3 & $\mathbf{7}$ \\
Plantaciones forestales & 18 & 54 & $\mathbf{7 2}$ \\
Aprovechamiento forestal & 6 & 9 & $\mathbf{1 5}$ \\
Aserradero & 3 & 6 & $\mathbf{9}$ \\
Tarimeras & 6 & 0 & $\mathbf{6}$ \\
Depósitos de madera & 7 & 5 & $\mathbf{1 2}$ \\
Mueblerías & 6 & 25 & $\mathbf{3 1}$ \\
\hline Total & $\mathbf{5 0}$ & $\mathbf{1 0 2}$ & $\mathbf{1 5 2}$ \\
\hline
\end{tabular}

Fuente: Murillo y Ávila (2014).

pmt: pulgada maderera tica. Unidad de medida de madera utilizada en Costa Rica. Corresponde a una pieza de una pulgada $\mathrm{x}$ una pulgada $\mathrm{x}$ cuatro varas $(2,54 \mathrm{~cm} \times 2,54 \mathrm{~cm} \times 3,36 \mathrm{~m}$ de longitud). En madera en rollo (en pie y en troza) y para diámetros de más de $60 \mathrm{~cm}$ un $\mathrm{m}^{3}$ equivale a 362 pmt; en madera aserrada un $\mathrm{m}^{3}$ equivale a 462 pmt. (Barrantes y Ugalde, 2017). 
Revista Universidad en DiÁlogo • Vol. 8, N. ², Julio-Diciembre, 2018, pp. 11-23

ISSN 2215-2849 • EISSN: 2215-4752

DOI: http://dx.doi.org/10.15359/udre.8-2.1

En la figura 2 se muestra la distribución geográfica de las personas incluidas en la encuesta, por distrito y por tipo de actividad.

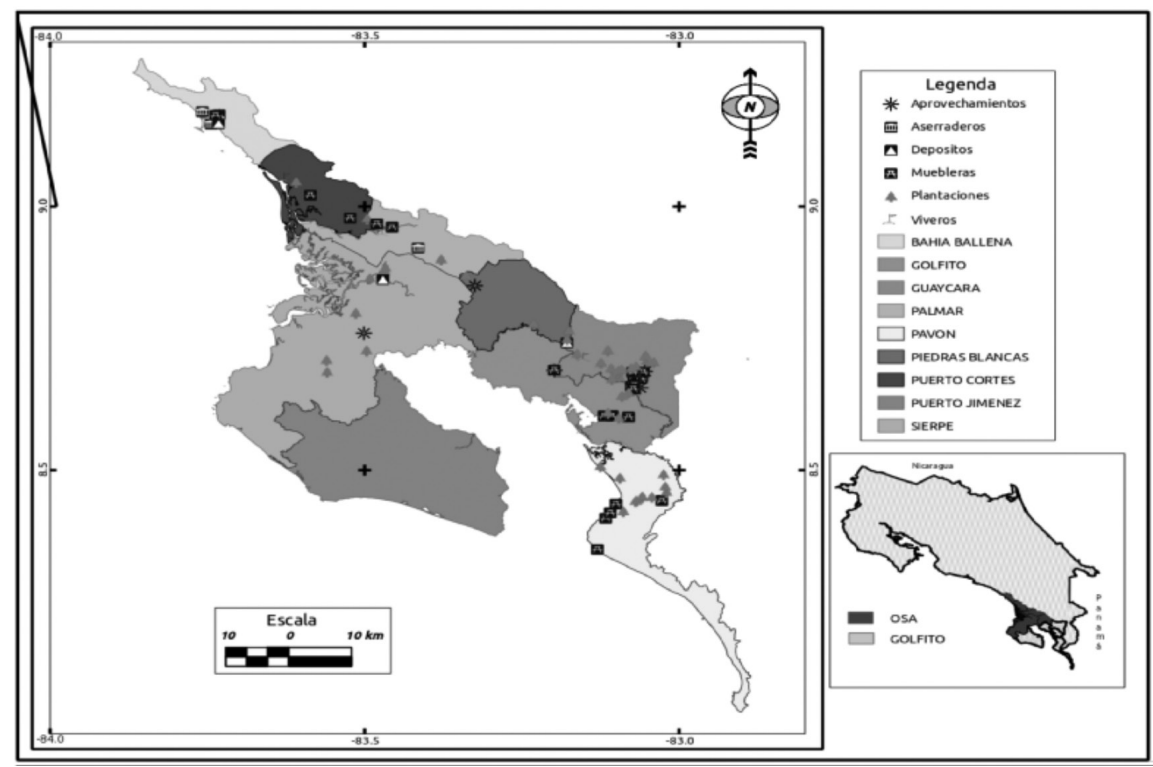

Figura 2. Distribución de las diferentes personas involucradas por distrito, según componentes de la cadena productiva.

Con el fin de incentivar la reforestación comercial en la zona, se estableció un vivero en La Palma de Puerto Jiménez de Golfito, en el cual se trabaja en la reproducción de material genético de alta calidad de especies forestales, como una fuente accesible para los/as productores/as de pequeña y mediana escala. El nexo con estudiantes de la UNA, con productores/as y empresarios/as, así como con instituciones relacionadas con el sector forestal costarricense, ha permitido que hoy en día los materiales distribuidos por el INISEFOR sean unos de los más solicitados por aquellas personas interesadas en lo que hemos denominado el cultivo eficiente e intensivo de madera.

En el cantón de Pérez Zeledón se realizaron diversas capacitaciones a mujeres y miembros de grupos organizados, así como a estudiantes de escuelas y colegios sobre temas relacionados con ambiente, cultivo de árboles, biodigestores y energías limpias; se promovió la instalación de 
huertas escolares y de viveros forestales y la reforestación con fines de protección en cuencas degradadas de la zona, así como el emprendedurismo rural como medio de sostenibilidad de las iniciativas.

Se trabajó en la microcuenca del río Águila, donde la mayoría de las propiedades son pequeñas, en un rango de 2 a 40 ha; las principales actividades económicas son ganadería extensiva y producción agrícola, principalmente frijol y maíz, así como yuca, plátano, café y arroz para subsistencia. Para este estudio se contó con el aporte económico de la cooperación noruega y uno de los indicadores que se analizó fue el uso de leña en los hogares, punto en el cual es interesante anotar que, dada la cobertura de electricidad con que cuenta el país, la leña no es la principal fuente energética, por lo que se logró determinar un consumo aproximado de entre 6 y $9,5 \mathrm{~m}^{3}$ por familia por año, lo cual contrasta con el resto de Centroamérica, donde cerca del $50 \%$ de la población utiliza la leña como fuente principal de energía y su consumo varía en un ámbito desde 11 a $29,4 \mathrm{~m}^{3}$ por familia por año. En esos países el abastecimiento de leña, aparte de plantear un reto a largo plazo, implica una serie de problemas en la salud, principalmente musculares, óseos y respiratorios.

\section{Impacto de la integración del quehacer universitario (investigación, extensión, docencia y producción)}

Uno de los factores básicos para el éxito en el establecimiento de plantaciones forestales es contar con excelente material de vivero, por lo que se trabajó con viveristas de los cuatro cantones involucrados sobre técnicas de identificación de fuentes semilleras, manipulación y germinación de la semilla, reproducción vegetativa de especies de rápido crecimiento y gestión general de los viveros forestales.

La actividad forestal necesita de un período de mediano a largo plazo para la obtención de los ingresos económicos finales, por lo que, en conjunto con los agricultores y las agricultoras, se establecieron sistemas productivos diversificados que permiten obtener rentas a corto plazo mientras se llega al turno final de corta, para lo cual se ensayó con la combinación de árboles con varias especies medicinales, comestibles y ornamentales (Murillo y Ávila, 2011).

Se compartieron las experiencias generadas mediante seminarios para el fomento del cultivo eficiente de la madera, en los cuales participaron 
ReVista Universidad en DiÁlogo • Vol. 8, N. ํㄹ 2, Julio-Diciembre, 2018, pp. 11-23

ISSN 2215-2849 • EISSN: 2215-4752

DOI: http://dx.doi.org/10.15359/udre.8-2.1

estudiantes, productores/as, académicos/as y profesionales de instituciones, tanto públicas como privadas. La organización de días de campo permitió la divulgación y capacitación sobre el cultivo intensivo de árboles, con productores/as, estudiantes, agricultores/as de la Zona Sur y de la Zona Norte y con autoridades de la UNA y de instituciones forestales del país.

También se propició la generación de capacidades en la población meta del proyecto, ligada a la producción forestal local, para lo cual se realizaron nueve talleres sobre emprendedurismo, iniciativas productivas y desarrollo local, con temas específicos como finanzas, cómo aprovechar los cambios, atreverse a soñar, cómo planear estratégicamente, contabilidad, entre otros. Para este trabajo se contó con el apoyo de estudiantes de la carrera de Administración del Campus Coto, Sede Brunca de la Universidad Nacional (Hernández, Ávila y Murillo, 2016).

A fin de mejorar los conocimientos de personas operadoras de aserraderos, en conjunto con el Instituto Nacional de Aprendizaje (INA) se elaboró y planificó el Curso Operación y Mantenimiento de Aserraderos Móviles, el cual finalmente, por asuntos fuera del control de los y las proyectistas, no fue posible impartir; sin embargo, el módulo se encuentra listo para ser dictado en cuanto se requiera.

Asimismo, varios/as estudiantes de la carrera de Ingeniería Forestal de la UNA elaboraron sus tesis de licenciatura y prácticas profesionales, las cuales han generado información útil para las personas propietarias de terrenos de la Zona Sur y para los/as productores/as interesados/as en la melina, la teca y especies nativas. Tal es el caso de aquellos trabajos que permitieron establecer metodologías y tratamientos adecuados para la siembra de melina (Ávila, Murillo, Murillo, Sandoval, 2015; Salas, Murillo, Ávila, Mata, 2016); el análisis del crecimiento de la teca en cercas o en bloques en fincas de los cantones de Osa, Corredores y Golfito (Granados, 2014), donde se definió que un 36\% del área total de dichos cantones presenta condiciones óptimas para el cultivo de la teca.

\section{Ligamen con instituciones del sector}

Contrariamente a lo sucedido en Costa Rica previo a los años ochenta, donde la mayor parte de la madera que se consumía en el país provenía de los bosques naturales, en los últimos años la principal fuente de esta materia 
prima han sido las plantaciones forestales. Según Barrantes y Ugalde (2016), un 77\% de la madera que se aprovechó en el 2015 provino de dicha fuente. De acuerdo con la Oficina Nacional Forestal (ONF), la principal especie maderable que se comercializa en Costa Rica es la melina, la cual es utilizada para la fabricación de material de embalaje, en construcción y para la fabricación de muebles (Barrantes y Ugalde, 2016). Por tales razones, se ha dado énfasis a la promoción del cultivo de dicha especie, de la cual se ha logrado producir materiales genéticos de gran valor y se han generado paquetes tecnológicos para su producción.

El nexo con instituciones y con la población busca que el cultivo de árboles se convierta en una actividad interesante, con buenos rendimientos para abastecer al mercado de la madera, mismo que en los últimos tiempos se ha visto disminuido en Costa Rica por distintos factores adversos, tales como una disminución en el uso de este tipo de recurso, dándose preferencia a los sistemas constructivos con hierro y cemento, un aumento en las importaciones de madera aserrada, principalmente pino chileno, una desmotivación general por la actividad forestal y la falta de un mayor desarrollo del sector industrial forestal que disminuye las posibilidades de competir, entre otros (Barrantes y Ugalde, 2016).

\section{Conclusiones}

El INISEFOR busca la construcción conjunta de una cultura forestal propia para los campesinos y las campesinas de regiones poco favorecidas del país, que les permita contar con actividades productivas armoniosas con el ambiente, diversificadas, que aporten los distintos servicios ecosistémicos, cercanas a sus tradiciones y, sobre todo, rentables.

En este período, los enlaces generados con finqueros/as, estudiantes de primaria, secundaria y universitarios/as, con organizaciones comunales, cooperativas, empresas privadas, instituciones estatales, otras universidades, entre otros actores, han permitido visualizar las necesidades existentes, la realidad de una clase campesina que en muchos casos cuenta con lo más importante: la tierra y el deseo de producir, pero carece de los insumos, de conocimiento técnico y financiero, del acceso a la agroindustrialización y a las cadenas de comercialización, por lo que en la mayoría de los casos se ve obligada a dejar en manos de los intermediarios sus cosechas y producción, ya sea ganado, madera o productos agrícolas. 
Revista Universidad en Diálogo • Vol. 8, N. ${ }^{\circ} 2$, Julio-Diciembre, 2018, pp. 11-23

ISSN 2215-2849 • EISSN: 2215-4752

DOI: http://dx.doi.org/10.15359/udre.8-2.1

Es real y tangible el posicionamiento logrado en la zona y para lograr finalmente cerrar el círculo y generar cambios sustanciales se requiere que, además de este acercamiento universidad-productores/as, exista un apoyo de instituciones, organizaciones y hasta un cambio de mentalidad que logre modificar poco a poco los patrones de producción poco productivos de la mayoría de finqueros/as de la zona.

La experiencia reafirma la necesidad de un trabajo interinstitucional, pues, aunque se logre demostrar que un determinado material genético de una especie forestal tiene los mejores desempeños, se adapta a la zona y es posible combinarla con las actividades agropecuarias, existe una serie de factores estructurales y coyunturales que impiden que los finqueros y las finqueras inviertan en reforestación, industrialización de la madera y comercialización de sus productos.

Los recursos invertidos permitieron llegar hasta la generación del conocimiento y experiencias piloto, no obstante, la tarea de proyectar, impulsar y replicar requiere sin lugar a dudas la confluencia y compromiso con varias instituciones del Estado, que son las llamadas a llevar a otra escala los resultados generados por estos proyectos.

\section{Referencias}

Ávila, C., Murillo, R., Murillo, O. y Sandoval, C. (2015). Desarrollo juvenil de clones de Gmelina arborea Roxb. de dos procedencias, en sitios planos del Pacífico Sur de Costa Rica. Revista Forestal Mesoamericana Kurú, 12(28), 23-35.

Barrantes, A. y Ugalde, S. (2016). Usos y aportes de la madera. Costa Rica. Estadísticas 2015. Oficina Nacional Forestal (ONF). San José, Costa Rica.

Barrantes, A. y Ugalde, S. (2017). Precios de la madera para las especies más comercializadas. Primer semestre del 2017. Recuperado de https://www. onfcr.org/media/uploads/documents/precios-de-la-madera-2017.pdf

Granados, M. (2014). Crecimiento de la teca (Tectona grandis Linn.f.) en plantaciones forestales y linderos maderables de la Zona Sur, Costa Rica. Tesis de Licenciatura en [...]. Escuela de Ciencias Ambientales. Universidad Nacional. Heredia, Costa Rica. 
Hernández, W., Ávila, C. y Murillo, R. (2016). Informe final del proyecto FIDA: paquete tecnológico para la producción de material vegetal de alta calidad en el establecimiento de plantaciones forestales de mangillo (Aspidosperma spruceanum) y melina (Gmelina arborea), como medio para fortalecer el desarrollo local en los cantones de Osa y Golfito. INISEFOR, Universidad Nacional. Heredia, Costa Rica.

Ministerio de Planificación Nacional y Política Económica (Mideplan). (2013). Índice de Desarrollo Social (IDS) 2013. Recuperado de https:// documentos.mideplan.go.cr/alfresco/d/d/workspace/SpacesStore/ ab677d6c-fafd-4128-86df-a6aa04ab70ef/IDS\%202013\%20resumen. pdf?guest=true

Murillo, R. y Ávila, C. (2011). Informe final de proyecto CONARE: mejoramiento de la capacidad productiva de pequeños y medianos reforestadores de la Zona Sur. Recuperado de www.inisefor.una. ac.cr/docs/Proyecto_Mejoramiento_Capacidad_Productiva Reforestadores_zona_sur_Informe_fiñal_resultados_2008_2010. pdf I2011

Murillo, R. y Ávila, C. (2014). Informe final de proyecto CONARE: manejo agroforestal participativo como inicio de encadenamientos productivos en fincas integrales. Recuperado de http://www.inisefor. una.ac.cr/docs/Proyecto_Manejo_Agroforestal_Participativo_en_ Fincas_Integrales_Informe_final_resultados_2010_2013.pdf

Salas, A., Murillo, O., Murillo, R., Ávila, C. y Mata, X. (2016). Evaluación de la severidad de la pudrición del tronco en Gmelina arborea Roxb. Revista Forestal Mesoamericana Kurú. Volumen especial (01-10). doi:10.18845/rfmk.v0i0.2547 